about diagnosis was mentioned in the text and no patient over age 30 had an $\varepsilon 4$ allele. For these reasons we chose not to include this study in our meta-analysis. However, as Prasher \& Haque point out inclusion of this study would have made little difference to our findings.

Whereas Prasher \& Haque rightly suggest that further research is needed to clarify the role of ApoE $\varepsilon 4$ in Alzheimer's disease in people with Down's syndrome, we were surprised to see that they have recommended ApoE genotyping as a possible screening test for dementia in this population. This will be totally inappropriate at this stage considering the uncertain relationship between Alzheimer's neuropathology and ApoE genotype in people with Down's syndrome, as we mentioned in the last paragraph of the Discussion in our paper.

We agree with Prasher \& Haque that the presence of $\varepsilon 4$ allele is neither necessary nor sufficient for the development of Alzheimer's disease.

Woolf, B. (1995) On estimating the relation between blood group and disease. Analysis of Human Genetics, 19 $251-253$

S. Deb, J.Williams \& M. J. Owen Division of Psychological Medicine, University of Wales College of Medicine, Heath Park, Cardiff CFI4 4XN

\section{Chronic fatigue syndrome and depression}

I found MacHale et al's (2000) discussion of their results confusing. According to the abstract and methods, they screened their patients with chronic fatigue syndrome (CFS) to exclude those with depression. Then they examined this group further using a standardised psychiatric interview (Schedule for Affective Disorders and Schizophrenia), in order to "exclude subjects with current psychiatric illness, with a particular emphasis on depression". The data from the Hamil- ton Rating Scale for Depression are difficult to interpret given the number of illnessrated items, but the scores did not indicate a significant degree of depression either. So, having excluded "subjects with depression or anxiety", why did the authors claim in their discussion that "the main limitation of the present study is that our CFS subjects had high levels of depression"?

If this is correct, why was their depression not picked up by the three measures? Why were these patients not excluded from the research as stated by the authors or, funds permitting, used as a comparison group (Costa et al, 1995; Fischler et al, 1998)? How depressed were the 10 patients on antidepressants and, if these were not effective, could their suboptimal treatment have contributed to their ongoing fatigue?

I was also baffled by the authors' suggestion that the thalamic hyperperfusion may reflect "increased attention to motor and cognitive tasks". What were the patients doing? The abstract states that the scans were conducted at rest. If the subjects had just completed a battery of cognitive tests, why did the authors not check to see whether the data available supported their hypothesis (Fischler et al, 1998)?

If this paper was subjected to peer review, why did no one query the selective discussion of the findings and the misrepresentation of the literature on CFS and psychopathology?

Costa, D. C., Tannock, C. \& Brostoff, J. (1995)

Brainstem perfusion is impaired in chronic fatigue syndrome. Quarterly Journal of Medicine, 88, 767-773.

Fischler, B., Flamen, P., Everaert, H., et al (1998) Physiopathological significance of ${ }^{99} \mathrm{mT}$ cHMPAO SPECT scan anomalies in chronic fatigue syndrome: a replication study. Journal of Chronic Fatigue Syndrome, 4, 15-30.

MacHale, S. M., Lawrie, S. M., Cavanagh, J.T., et al (2000) Cerebral perfusion in chronic fatigue syndrome and depression. British Journal of Psychiatry, 176, 550-556.

E. Goudsmit 23 Melbourne Road, Teddington, MiddlesexTWII 9QX
Authors' reply: As explained in the method section, the potential participants were screened by excluding those scoring above case threshold in the Hospital Anxiety and Depression (HAD) scale, a self-rating scale that does not require a detailed interview. The remaining participants were then interviewed using the Schedule for Affective Disorders and Schizophrenia to further exclude any current mental illness.

First, in the discussion we say: "The main limitation of the present study is that our CFS subjects had high levels of depression: almost half were on psychotropic medication and five had a previous history of depression". "Had high levels of depression" is defined by what follows after the colon. There is, therefore, no contradiction. Participants were not currently depressed, but some were receiving antidepressant medication and some had previously been depressed.

Second, regarding that point made relating to our comment that "thalamic overactivity in CFS (and depression) may, therefore, reflect increased attention to motor and cognitive tasks ...". The perceived contradiction is that participants were at rest during uptake of the tracer, i.e. not currently engaged in motor or cognitive tasks. It is clearly speculative that increased thalamic activity at rest will also mean increased thalamic activity during tasks. What was implied, however, was that increased baseline or resting activity of the thalamus may be an underlying brain marker that is related to patients being more attentive to motor and cognitive activity, as they occur.

S. M. MacHale, S. M. Lawrie, J. T. Cavanagh, M. F. Glabus, C. L. Marray, K. P. Ebmeier Department of Psychiatry and MRC Brain Metabolism Unit, University of Edinburgh, Kennedy Tower, Royal Edinburgh Hospital, Morningside Park, Edinburgh EHIO 5HF

G. M. Goodwin University Department of Psychiatry, Warneford Hospital, Oxford OX3 7JX 